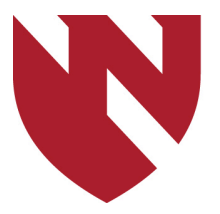

\title{
Minimizing Psychological Distress and Promoting Resilience During Quarantine: Piloting the Town Hall Model
}

David S. Cates

Nebraska Medicine, dcates@nebraskamed.com

Tell us how you used this information in this short survey.

Follow this and additional works at: https://digitalcommons.unmc.edu/tnmc_articles

Part of the Psychology Commons

\section{Recommended Citation}

Cates, David S., "Minimizing Psychological Distress and Promoting Resilience During Quarantine: Piloting the Town Hall Model" (2021). Journal Articles: Nebraska Medicine. 5.

https://digitalcommons.unmc.edu/tnmc_articles/5

This Article is brought to you for free and open access by the Nebraska Medicine at DigitalCommons@UNMC. It has been accepted for inclusion in Journal Articles: Nebraska Medicine by an authorized administrator of DigitalCommons@UNMC.For more information, please contact digitalcommons@unmc.edu. 


\title{
Minimizing Psychological Distress and Promoting Resilience During Quarantine:
}

\section{Piloting the Town Hall Model}

\author{
David S. Cates \\ Department of Psychiatry, Nebraska Medicine / University of Nebraska Medical Center
}

\section{Author Note}

DAVID S. CATES received his PhD in clinical psychology from the University of Kansas. He is the Director of Behavioral Health at Nebraska Medicine and Vice Chair of Clinical Operations in the Department of Psychiatry at the University of Nebraska Medical Center. Dr. Cates also serves as the behavioral health consultant to the Nebraska Biocontainment Unit and the National Quarantine Unit. His areas of professional interest include the psychological effects of medical isolation and quarantine, promoting resilience in healthcare workers, and child clinical psychology.

The author thanks the administration, staff and physicians of the National Quarantine Unit, Nebraska Biocontainment Unit and Davis Global Center for Health Security whose visionary leadership and dedication to excellence made this work possible, in particular, Shelly Schwedhelm, MSN, RN, NEABC, Angie Vasa, MSN, RN, Kate Boulter, BS, RN, Morgan Shrader, BSN, RN, James Lawler, MD, Angela Hewlett, MD, Chris Kratochvil, MD, and Mike Wadman, MD. The author owes a special debt of gratitude to Ted Cieslak, MD, for participating in the majority of the Town Hall meetings, during which he displayed unwavering patience, compassion and humor. The author is additionally grateful to Leah Cates for her expert editorial assistance.

The Office of Regulatory Affairs at the University of Nebraska Medical Center determined that this project did not constitute human subjects research as defined by 45CFR46.102.

Correspondence concerning this article should be addressed to David Cates, PhD, Nebraska Medicine, 984185 Nebraska Medical Center, Omaha, NE 68198-4185, United States. Email: dcates@nebraskamed.org. 


\begin{abstract}
Quarantine and isolation are important public health strategies for containing highly hazardous communicable disease outbreaks, particularly when vaccines and effective treatments are unavailable. Despite their effectiveness in disease containment, both quarantine and isolation, whether home- or facility-based, can lead to negative psychological outcomes in the short and long term. This article describes a novel, evidence-informed pilot intervention to prevent psychological deterioration during facility-based quarantine and isolation. Designed for the National Quarantine Unit during the repatriation of 15 American passengers exposed to COVID-19 on a cruise ship, the model incorporates findings from several areas of research, including factors that increase stress during quarantine and isolation, resources and competencies thought to contribute to individual resilience, and the role of social support in buffering stress. The high participation rate, in connection with positive feedback from guests, suggests that the pilot intervention holds promise for mitigating the potentially damaging psychological effects of facility-based quarantine and isolation.

Keywords: quarantine, isolation, COVID-19, infection control, resilience
\end{abstract}

\title{
Public Significance Statement
}

This case study describes a novel, pilot intervention to prevent psychological deterioration in a group undergoing facility-based quarantine after exposure to COVID-19. The intervention addressed participants' basic needs and comfort, provided them with timely information about the disease, and introduced strategies believed to enhance resilience. Qualitative feedback suggests the model holds promise for mitigating the potentially negative psychological consequences of quarantine, though controlled research is necessary to test this hypothesis. 


\section{Minimizing Psychological Distress and Promoting Resilience During Quarantine: \\ Piloting the Town Hall Model}

Quarantine and isolation are critical public health response strategies during highly hazardous communicable disease (HHCD) outbreaks, particularly when vaccines and effective treatments are not available. Quarantine involves confining and monitoring individuals with a high-risk exposure to a disease but not a confirmed diagnosis. For those who do not contract the illness, the duration of quarantine is the presumed incubation period for the infectious disease of concern. Isolation, in contrast, entails segregating individuals with the disease from those without it, lasting as long as the individual remains infectious. Isolation for infectious illnesses frequently occurs in healthcare settings, whereas quarantine is often completed at home. However, quarantine in a facility, such as a hospital or a government-funded quarantine center, may be necessary in a variety of situations, such as homelessness, inability to follow quarantine guidelines, a small living space shared with one or more immunocompromised or otherwise vulnerable people, a novel pathogen, arrival from a foreign country, and when transportation home poses risks (e.g., Centers for Disease Control and Prevention, 2003a, 2003b; Jordan-Martin et al., 2020). Both quarantine and isolation, whether at home or in a facility, place individuals at risk for psychological distress. The current paper describes an intervention designed to enhance resilience and prevent negative psychological consequences in a group undergoing a facilitybased quarantine and isolation.

\section{Psychological Effects of Quarantine and Isolation}

Individuals in quarantine and isolation are at risk for negative psychological outcomes due not only to the conditions prompting their implementation but also the nature of the quarantine/isolation itself. With both home-based quarantine and facility-based isolation come a loss of control associated with confinement to a defined space, sometimes for an unknown time. There may be financial strain due to loss of income (e.g., Blendon et al., 2004; Cava et al., 2005; Desclaux et al., 2017; DiGiovanni et al., 2004; 
Jeong et al., 2016; Reynolds et al., 2007); difficulty meeting family and social obligations (e.g., Desclaux et al., 2017; DiGiovanni et al., 2004; Reynolds et al., 2007); limitations in the ability to engage in valued activities, including socialization (e.g., Barratt et al., 2011; Hawryluck et al., 2004; Knowles, 1993); and diminished sensory input (e.g., Ward, 2000). In addition, the typically rapid and unexpected implementation of quarantine and isolation may itself be stressful (Cava et al., 2005; Madeo, 2001). The research on isolation focuses predominantly on patients in hospital settings, whereas studies of quarantine concentrate primarily on home quarantine. Both areas of research are reviewed briefly below.

The literature on isolation for infectious illness suggests that compared to hospitalized patients who are not isolated, patients treated in isolation are at risk for symptoms of depression and anxiety (e.g., Catalano et al., 2003; Day et al., 2011; Day et al., 2013; Gammon, 1998; Soon et al., 2013; Tarzi et al., 2001). Research with patients isolated for multidrug-resistant organisms or C. Difficile suggests that symptoms are likely to emerge after 2-3 days of isolation and may intensify with increased length of treatment (Cates et al., 2018). In addition, qualitative-phenomenological studies of patients in medical isolation suggest that they may experience loneliness (e.g., Newton et al., 2001), boredom (e.g., Oldman, 1998), fear (e.g., Barratt et al., 2011; Guillemin et al. 2014), alienation (e.g., Skyman et al., 2010) and stigmatization (e.g., Barratt, 2011; Madeo, 2001). There are also reports of patients behaving disruptively, including venting anger and throwing objects at staff (Mayho, 1999), seeking assistance with tasks they are able to accomplish independently, and leaving the isolation room without permission (Oldman, 1998).

Similarly, a high prevalence of psychological distress has been reported among individuals in homebased quarantine, including symptoms of posttraumatic stress (e.g., Bai et al., 2004; Hawryluck et al., 2004; Reynolds et al., 2007; Sprang \& Silman, 2013; Wu et al., 2009), depression (e.g., Hawryluck et al., 2004; Liu et al., 2012) and anxiety (e.g., Desclaux et al., 2017; DiGiovanni et al., 2004; Jeong et al., 2016). 
As with medical isolation, quantitative and qualitative-phenomenological studies of quarantine reveal that individuals report experiencing loneliness (e.g., DiGiovanni et al., 2004; Reynolds, et al., 2007), boredom (e.g., DiGiovanni et al., 2004; Reynolds et al., 2007), isolation (Cava et al., 2018; DiGiovanni et al., 2004; Hawryluck et al., 2004; Reynolds et al., 2007), anger (e.g., Jeong et al., 2016) and stigma (Caleo et al., 2018; Cava et al., 2018; Desclaux et al., 2017; DiGiovanni et al., 2004; Hawryluck et al., 2004). Factors that may increase stress and fear during quarantine include insufficient supplies and inadequate, confusing and/or poorly coordinated information from public health authorities (Brooks et al., 2020).

In contrast to studies reporting significant psychological distress among individuals in home quarantine, research with U.S. military personnel deployed to West Africa to provide logistical support during the Ebola crisis found minimal mental health concerns among the 501 study participants forced to undergo a 21-day quarantine in a controlled monitoring area (CMA) on a military base upon their return (Adler et al., 2018). In this study, attitudes toward quarantine were generally favorable, with 42.7\% agreeing that quarantine was a good idea, $52.5 \%$ concurring that it would help keep the community safe, and $71.8 \%$ acknowledging that quarantine would reduce anxiety in the community. The investigators found that health-promoting leadership behaviors, such as encouraging emotion regulation and self-care for those in quarantine, were associated with more positive attitudes toward quarantine and fewer depression and anxiety symptoms. In addition, family support was associated with less functional impairment and more positive attitudes toward quarantine among service members. The authors speculated that providing families with a clear rationale for quarantine may have increased acceptance of their loved one's confinement to the CMA.

The evolving literature on isolation and quarantine, summarized above, informed development of the operating standards for the National Quarantine and Nebraska Biocontainment Units at the University of Nebraska Medical Center, where the current intervention was developed and piloted. 


\section{The National Quarantine Unit (NQU) and Nebraska Biocontainment Unit (NBU)}

In 2016, the U.S. Department of Health and Human Services awarded a grant to the University of Nebraska Medical Center and its affiliated clinical enterprise, Nebraska Medicine, to develop a Training, Simulation and Quarantine Center. In addition to supporting education and training for federal health care and public health service personnel to care for patients with HHCDs, the grant funded development of a National Quarantine Unit (NQU) to monitor individuals and groups exposed to HHCDs. The opening of the NQU in January, 2020 followed the creation of the Nebraska Biocontainment Unit (NBU) in 2005. The NBU, commissioned by the United States Centers for Disease Control and Prevention, was designed to treat people affected by bio-terrorism and hazardous communicable diseases. The NBU, which cared for several patients medically evacuated from West Africa with Ebola Virus Disease in 2014, is one of ten Regional Emerging Special Pathogen Treatment Centers in the United States.

The nurses, respiratory therapists and patient care technicians who staff the NQU and NBU are members of the same team who normally work on units throughout the hospital, receive ongoing education and training in treating patients with HHCDs, and stand ready to activate with the NQU/NBU at any time. For the joint NQU/NBU activation described below, several individuals were cared for in both units at various times depending on the clinical course of their illness.

\section{Psychological Considerations}

The NQU, like the NBU, included behavioral health considerations from its inception, in recognition of the fact that quarantine and isolation pose psychological risks, as outlined above. These considerations were translated into standard operating procedures for the NQU. Based in part on the Adaptable Quarantine Procedures Manual (New York City Department of Health and Mental Hygiene, 2010), these procedures include informing quarantined individuals about available activities, supplies, books and magazines; providing access to exercise equipment, television and internet; problem-solving around desired activities not readily available; facilitating contact with family members and friends, 
either virtually or in-person, as appropriate based on the index disease and route of transmission; identifying and honoring preferences regarding visitors (consistent with infection control protocols); encouraging quarantined individuals to plan daily routines; ensuring opportunities to practice religion; providing clear and consistent communication about the index disease and rationale for infection control procedures; using active, empathic listening; and offering consultation with a behavioral health professional.

\section{Role of the Behavioral Health Consultant}

Both the NQU and NBU have a behavioral health consultant (BHC) who, since 2015, has been a clinical psychologist (the author). The BHC's responsibilities include the following: 1) Coordination of behavioral health services for guests/patients, including individual consultation as needed; 2) Consultation to unit leadership and staff regarding prevention and management of psychiatric disorders and behavioral health concerns that may arise among guests/patients; 3) Support for staff through ongoing resilience training (teaching staff to self-identify signs of stress, use relaxation techniques, increase social support, challenge distorted thoughts, etc.) and 1:1 assistance when requested. ${ }^{1}$

\section{The Town Hall Model}

\section{Impetus for Development}

On February 17, 2020, 13 passengers quarantined on a cruise ship docked in Yokohama, Japan, due to an outbreak of novel coronavirus disease (COVID-19) were evacuated to the NQU at the University of Nebraska Medical Center / Nebraska Medicine. ${ }^{2}$ Prior to their evacuation, 11 passengers tested positive

${ }^{1}$ The staff support role is included due to the challenges inherent in treating patients who have, or have been exposed to, HHCDs. These challenges include fear of contracting the illness, worry about the safety of family members, being separated from usual workgroups when the units are activated, and proper use of personal protective equipment (PPE) among others (for more information, see Cates et al., 2018).

${ }^{2}$ COVID-19 is a contagious respiratory illness with a wide range of symptoms that may include fever, cough and shortness of breath. Although most cases are mild, research suggests that between $14 \%$ and $19 \%$ require hospitalization and between $3 \%$ and $5 \%$ intensive care, with risk for severe disease increasing with age and underlying health conditions (Centers for Disease Control and Prevention, 2020). As of December 21, 2020, the case fatality rate in the United States was $1.8 \%$ (Coronavirus Resource Center, 2020). 
for severe acute respiratory syndrome coronavirus 2 (SARS-CoV-2), the virus that causes COVID-19, and two tested negative. Two additional evacuees who tested positive for SARS-CoV-2 were transferred from facilities in Texas and California on the evenings of February 24 and 25, respectively. Both were spouses of one of the original 13 guests, bringing the total number to 15.

For the 13 guests who tested positive for SARS-CoV-2, release was dependent on three negative polymerase chain reaction (PCR) tests, based on samples from nasopharyngeal swabs, 24 hours apart. For the two individuals who tested negative, the release date was 14 days -the presumed incubation period of COVID-19-from the negative test result obtained just prior to arrival at the NQU, as long as they did not develop symptoms or test positive prior to release. These evacuees were under significant stress because they already had been quarantined for two weeks on the cruise ship; the length of time quarantined/isolated in Nebraska was indeterminate; they had, or were at risk of developing, a lifethreatening disease; and they were subject to the additional stressors attendant to confinement described above under Psychological Effects of Quarantine and Isolation. As a result, the NQU team began exploring ideas beyond the standard operating procedures outlined above to help the passengers manage stress, enhance resilience and prevent psychological deterioration.

The NQU/NBU team was not aware of any controlled trials of interventions designed to mitigate the potentially negative psychological consequences of quarantine/isolation. In the absence of such data, the team considered evidence from several lines of research, including factors that increase stress during quarantine and isolation, resources and skills associated with resilience, the role of social support in buffering stress, the effects of relaxation training on psychological distress, and therapeutic factors in group psychotherapy. In addition, several team members had recently been involved in a 14-day quarantine of 57 Americans returning from China. This quarantine, which took place at the Camp Ashland Nebraska National Guard training site, was led by the US Centers for Disease Control and Prevention (CDC) and the US Department of Health and Human Services Office of the Assistant Secretary 
for Preparedness and Response (ASPR). During this quarantine, CDC personnel along with Family Child Case Workers from ASPR held daily "Town Hall" briefings with those in quarantine to address protocols, rules and timelines for quarantine as well as day-to-day needs, such as meals, activities and supplies.

Using this model as a framework and incorporating the research mentioned above, the team developed the NQU Town Hall intervention for facility-based quarantine. The NQU Town Hall was a daily audio-only teleconference during which medical, nursing, case management and behavioral health staff shared information with guests, invited them to ask questions, and encouraged interaction with one another. The goals and objectives are outlined below, followed by a detailed description of the meeting structure and contents.

\section{Goal, Objectives and Evidence Base}

The goal of the Town Hall was to mitigate the potentially negative psychological consequences of quarantine/isolation. The objectives in support of this goal, their operationalization, and the associated evidence base are described below.

\section{Objective 1}

Promote guests' trust and confidence in staff by providing timely and accurate information about the SARS-COV-2 virus and COVID-19, including the course of the illness, trends in prevalence and mortality, means of transmission, and testing requirements for rescinding quarantine orders.

A recent literature review of the psychological effects of quarantine suggests that when public health officials lack transparency or provide inadequate and/or confusing information, those in quarantine are likely to experience increased stress (Brooks et al., 2020). In a sample of 129 residents of Toronto, Canada, quarantined during the SARS outbreak, for example, 30\% reported receiving inadequate information about SARS (Hawryluck et al., 2004). These individuals expressed anger about the incomplete information, frustration that officials were difficult to reach, and anxiety about insufficient communication regarding routes of disease transmission and the course of illness. Similarly, 
in a study of quarantine for Ebola exposure in Dakar, Senegal, Desclaux et al. (2017) found that nearly all of the 70 individuals interviewed about their quarantine experience reported anxiety-induced insomnia due to inadequate information about their level of risk. In contrast, the study of US military personnel quarantined after their deployment to West Africa (Adler et al., 2018), described above, suggests that providing a clear rationale for quarantine and communicating frequently about self-care may be associated with better mental health outcomes and a more positive view of the experience. Based on the above findings, the Town Hall promoted daily, transparent communication with the medical, nursing and behavioral health teams, including opportunities for guests to ask questions.

\section{Objective 2}

Address concerns and requests pertaining to daily needs and comfort on the unit, including dietary preferences, supplies and internet connectivity.

Difficulty accessing basic supplies, such as food, medicine, clothes and internet connectivity, is a potential source of distress for those in quarantine (Brooks et al., 2020). For example, in a study of 1,656 residents of South Korea quarantined for exposure to Middle East Respiratory Syndrome (MERS) in 2015, Jeong et al. (2016) found that having inadequate supplies during quarantine increased risk for anxiety and anger four to six months after release. Both the Service Access Team (a U.S. government case management resource; additional information below) and NQU/NBU nursing staff ensured that guests had access to needed supplies as part of standard operating procedures. The Town Hall reinforced the availability of such assistance by incorporating a formal daily check-in regarding guests' needs and comfort on the unit.

\section{Objective 3}

Enhance skills, behaviors and resources thought to contribute to resilience.

Resilience may be conceptualized as "the capacity of a dynamic system to adapt successfully to disturbances that threaten system function, viability, or development" (Masten, 2014, p. 6). On an 
individual level, resilience can be construed as the ability to adapt to, or bounce back from, challenging situations (e.g., Meichenbaum, 2012). Thoughts and behaviors associated with individual resilience include focusing on positive emotions and experiences, cultivating gratitude, finding meaning and growth in adversity, following a routine, keeping physically fit, and effectively managing stress (e.g., Meichenbaum, 2012; Park et al., 2015). Connecting with social supports, addressed in Objective 4 below, is another important component of individual resilience (e.g., Park et al., 2015; Southwick et al., 2014). In addition, it is well established that mindfulness meditation and formal relaxation practices can reduce symptoms of anxiety, depression and other dimensions of psychological distress (e.g., Goyal et al., 2014; Li et al., 2020; Kim \& Kim, 2018; Walsh, 2011; Zech et al., 2017). Based on these findings, the Town Hall included information and training on the thoughts, behaviors and skills identified above.

\section{Objective 4}

Increase social support by fostering a sense of community ${ }^{3}$ and cohesiveness among guests, and by encouraging and facilitating connection with outside social supports.

Although this objective was informed by the robust literature relating social support to physical and mental well-being (e.g., Holt-Lunstad et al., 2017), its operationalization borrowed heavily from the literature on group psychotherapy. Yalom and Leszcz (2005) describe how group leaders influence norms in psychotherapy groups by establishing rules, modeling behaviors, and subtly and explicitly reinforcing actions that conform to the desired group culture. Although not leading a psychotherapy group per se, the BHC employed these strategies in the Town hall to encourage interaction, feedback, and a supportive and accepting environment among participants. Furthermore, the BHC worked to establish a sense of community and solidarity by promoting several therapeutic factors identified by Yalom and Leszcz (2005) as elemental change agents underlying effective groups, namely, universality,

\footnotetext{
${ }^{3}$ Sense of community can be defined as "a feeling that members have of belonging, a feeling that members matter to one another and to the group, and a shared faith that members' needs will be met through their commitment to be together" (McMillan \& Chavis, 1986, p. 9).
} 
altruism, instillation of hope, imparting of information, and group cohesiveness. Although they operate interdependently (Yalom \& Leszcz, 2005), separate strategies were developed to promote each therapeutic factor. Table 1 shows the five therapeutic factors and associated Town Hall interventions. Similar strategies were successfully incorporated in a mandatory, time-limited support group for 12 college students in Taiwan immediately after their experience with quarantine during the SARS outbreak (Pan et al., 2005).

In addition, by promoting daily interaction among the guests themselves, as well as between guests and staff, the Town Hall was designed to prevent feelings of loneliness, fear, isolation, boredom and stigmatization, all of which have been reported in the literature on both quarantine and isolation (e.g., Barratt et al., 2011; Caleo et al., 2018; Cava et al., 2018; Desclaux et al., 2017; DiGiovanni et al., 2004; Guillemin et al., 2014; Hawryluck et al., 2004; Madeo, 2001; Newton et al., 2001; Oldman, 1998; Pan et al., 2005; Reynolds, et al., 2007; Skyman et al., 2010).

\section{Participants}

All repatriated cruise ship passengers brought to the NQU/NBU in February 2020 were invited to participate in the Town Hall meetings. They were American citizens ranging in age from 54 to 80 (mean $=69.7, S D=7.4)$. There were eight females and seven males. They lived in cities throughout the United States. Five were originally from East or Southeast Asia and one Western Europe, with English as the second language for these six individuals. All but one were fluent in English; for the one passenger not fluent in English, their spouse assisted with interpretation. All passengers had a spouse in quarantine/isolation in the NQU/NBU except one whose spouse was quarantined in another state. One couple shared a room in the quarantine unit. All others were in separate rooms. On March 7, 2020, 3 weeks after the first passengers arrived and at the midpoint of the Town Hall meetings, a local healthcare worker, a female in her 20s, joined the group. She had been exposed to COVID-19 in the community and was unable to quarantine at home. This brought the total number to 16 . 
Technically, the three SARS-CoV-2 negative individuals were in quarantine, and the 13 SARS-CoV-2 positive individuals were in isolation, consistent with the definitions above. The majority of the passengers had minimal or no symptoms of COVID-19 and did not receive medical treatment. Twelve were monitored in the NQU throughout their time at Nebraska Medicine. Four were transferred to the NBU for treatment during their stay due to progressive shortness of breath, palpitations and/or extreme weakness. Some were discovered to be hypoxic and others had new-onset cardiac arrhythmia or worsening of a known chronic neurologic or cardiac condition. The average length of stay in the NBU was 9 days. All were managed with supportive care and all survived.

Individuals quarantined in the NQU are considered "guests" and are not registered as patients". Individuals treated in the NBU, in contrast, are registered as patients of Nebraska Medicine. For simplicity, all individuals cared for during this activation will be referred to as "guests" and their time in the NQU/NBU as "quarantine." Four of the guests met individually with the BHC for one or more sessions, concurrent with the Town Halls, to address psychological distress related to quarantine.

Each Town Hall included the BHC, at least one physician from the NQU/NBU medical team, at least one nurse from the NQU/NBU team, and a Service Access Team consisting of two United States Public Health Service Commissioned Corps officers who provided case management.

\section{Meeting Frequency and Venue}

The inaugural Town Hall meeting was held on February 25, 2020, one week after the arrival of the initial 13 passengers. Ideally, a daily meeting with guests and unit leaders would have started immediately; however, as there was minimal notice for this inaugural NQU activation, along with parallel activation of the NBU, the priorities during week 1 were staffing of the two units, logistics regarding passenger medical care and basic needs, and establishing core groups for internal and regional

\footnotetext{
${ }^{4}$ Because they are not registered as patients, guests in quarantine do not receive any of the standard behavioral health or daily functioning measures usually administered at intake.
} 
incident command meetings that included many of the NQU and NBU team members. As the week progressed, the need to prioritize a structured venue in which to share information and coping resources became increasingly clear based on questions and requests from guests.

Town Halls were convened daily, weekends included, until the last passenger left on March 18, 2020. In all, the group met 22 times. Because guests were quarantined separately from one another, the Town Halls were held virtually. While the original intention was to have synchronous audio and video communication, some guests could not access the video connection due to limitations with, or separation from, their preferred communication devices. ${ }^{5}$ As a result, the meetings were conducted as audio-only Zoom teleconferences, with guests in their rooms and most staff members in a single conference room, though at times in separate locations. Participation was voluntary. Each meeting lasted approximately 40-45 minutes and followed the structure below.

\section{Meeting Structure}

\section{Introduction}

After welcoming guests and staff to the meeting, the BHC announced which staff members were present, took guest attendance, described the purpose of the Town Hall, and reviewed meeting guidelines. The BHC explained that the reasons for meeting were to share information about quarantine and the latest developments regarding COVID-19, answer guests' questions, and provide information and resources for managing stress related to the quarantine experience. Meeting guidelines included muting phones when not speaking and keeping other guests' information private. After the first week, the introduction was abbreviated, generally consisting of the BHC's stating who was in the room and taking attendance of those not physically present so that all participants knew who was on the call.

\footnotetext{
${ }^{5}$ The NQU budget included funds for just one set of tablets for communication between service providers not using personal protective equipment (PPE) and guests. Consequently, the NQU was not able to provide audiovisual communication devices for guest use during Town Hall meetings.
} 


\section{Medical Update}

A physician from the NQU/NBU provided an update on the pandemic, including the number and location of new and cleared cases nationally and internationally, current thinking about the degree of contagion and modes of transmission, course and severity of the illness, and criteria for release from quarantine. Guests were invited to ask questions, and the physician took as much time as necessary to answer each one. Queries were wide-ranging, including how long it takes to recover from the illness, the likelihood that a state authority might require another round of quarantine following federal quarantine, whether they might be transferred to another facility, how someone can test positive after previously testing negative, what to do if they thought it was time for their next SARS-CoV-2 test but had not been approached, whether they would receive a formal document indicating they had been cleared, and clarification regarding content they read on the internet. Personal medical questions, in particular concerns that required more lengthy evaluation or physical assessment, were noted and addressed in individual follow-up meetings with the medical providers.

During the first week of Town Hall meetings, the medical update and subsequent question-andanswer period typically lasted from 20 to 25 minutes. As the Town Halls progressed, guests asked fewer questions, and this portion of the meeting generally lasted between 10 and 15 minutes.

\section{SAT Team Update}

Service Access Teams ("SAT Teams") are groups of United States Public Health Service Commissioned Corps officers who assist with the health and case management needs of individuals affected by presidentially-declared disasters and public health emergencies. During the course of the current quarantine, three 2-person teams serving sequentially helped the cruise ship passengers resolve logistical challenges, including retrieving luggage, working with the cruise company on travel reimbursement, purchasing personal care items, and providing transportation to the airport upon release from quarantine. In the Town Hall, the SAT team provided updates on the above logistical items 
and responded to questions from guests. For the first ten meetings, SAT team updates typically lasted 13 minutes. Thereafter, SAT team members attended, but they generally did not provide updates and guests did not ask questions, as most of the SAT team's work at that point was unique to individual passengers and completed outside of the Town Hall meetings.

\section{Nursing Team Update}

During this portion of the Town Hall, nurses were available to address concerns about activities of daily living on the unit, such as food options, internet connectivity, garbage pickup and cleaning supplies. In the first week, this segment of the meeting typically lasted 3-5 minutes, as guests inquired about and made requests regarding basic needs, with a focus on food choices. In particular, several guests expressed a desire for more fruits and vegetables and fewer calorie-dense items. Once the menu was adjusted in response to guest preferences, the nursing update generally lasted less than a minute, with nurses checking in to ask about needs, and guests typically raising no issues but often expressing gratitude for the nurses' responsiveness, compassion and professionalism.

\section{Resilience and Wellness Training}

During each Town Hall, the BHC shared information on a topic related to resilience or wellness and then facilitated a discussion on that topic, often including an in-session exercise. Topics included creating a daily routine, using formal relaxation exercises (viz., deep breathing, body scan and visualization), developing positive coping statements, finding meaning and growth in the quarantine experience, accessing social support, cultivating gratitude, practicing mindfulness, engaging in physical exercise, restructuring distorted thoughts, using cognitive-behavioral interventions for insomnia, handling interview requests from news media, and anticipating sources of stress and feelings they might experience upon returning home, including emotional and behavioral signs that may indicate a need for professional help. (Table 2 in the supplemental online material shows the primary resilience and 
wellness topic for each Town Hall meeting.) With regard to the relaxation and mindfulness skills, the $\mathrm{BHC}$ led in-session exercises generally lasting from three to five minutes.

After presenting information and, when relevant, leading a formal mindfulness or relaxation exercise, the BHC engaged participants in a discussion about how the skills and ideas related to their lives and how they might employ them during quarantine and thereafter. In addition, at one point during many of the Town Hall meetings, typically after the resilience and wellness discussion, the BHC asked a "question of the day" to promote a sense of community. The questions ranged from "ice breakers" (e.g., "Share one thing about yourself, such as where you are from, something about your family, or a hobby") to ways of coping with quarantine (e.g., "Share something you are doing to keep busy"; "Share some way in which you have grown or made a positive change as a result of this experience").

After each Town Hall, the BHC, in consultation with the medical, nursing and SAT teams, as needed, created a written summary of the meeting contents. The write-up often included additional resources connected to the resilience and wellness topic, such as mindfulness exercises, recommended relaxation apps, suggested coping self-statements, and ways to keep busy. Unit nurses delivered the written summaries to guests with dinner that evening or with breakfast the following morning.

As the duration of the medical, SAT and nursing portions of the Town Hall decreased, the length of the resilience and wellness segment increased, allowing for more in-depth exploration of the day's topic and additional time for guests to share their experiences.

\section{Guest Responses to the Town Hall}

Because the Town Hall was developed in response to a rapidly evolving situation and not in connection with a formal research program, there were no baseline or follow-up measures of psychological or adaptive functioning and no control group, making evaluation of the intervention 
challenging. However, two sources of data-participation rate and feedback from guests-provide insight into the effectiveness of the intervention.

\section{Participation Rate}

Because attendance was voluntary, one measure of the Town Hall's success is the percentage of guests who elected to attend. Figure 1 shows the number of guests in quarantine and the number who attended the Town Hall throughout the period of time the intervention was offered. As seen in Figure 1, the total number of guests varied throughout the NQU/NBU activation. The initial increase from 14 to 15 resulted from the transfer of a cruise ship passenger quarantined in another state to join their spouse in Nebraska. The dip in attendance on February 26 and February 27 was due to a miscommunication regarding the Town Hall Zoom meeting ID, resulting in several guests' being unable to join. On March 1, the two SARS-CoV-2 negative individuals in quarantine, as well as two previously positive guests who met testing criteria for release, departed the unit for home. Three additional guests met criteria for release on the following three days, leaving eight of the original 15. On March 7, the local healthcare worker who had been exposed to COVID-19 and who was unable to quarantine at home joined the group, bringing the total number to nine. The remaining guests were cleared for release at various points beginning March 10, with the final guest departing on March 18 after a total of 30 days in quarantine in the NQU, following two weeks in quarantine on the cruise ship.

The overall rate of participation was $82 \%$. Approximately half of the gap between the total number of guests and Town Hall attendance was due to one guest who, beginning March 3, spent most of the time in the NBU for treatment of COVID-19 symptoms and who did not feel well enough to participate, along with this guest's spouse who, as days passed, elected to spend more time (virtually) with their spouse rather than attend the Town Hall. Much of the remaining gap was a combination of the aforementioned miscommunication regarding the Zoom meeting ID, guests preparing for departure on their final day, and, per their report, guests' napping or forgetting. A comparison of attendance for the 
first 11 days (86\%) with that of the second 11 days (73\%) suggests that interest remained high throughout the period of quarantine, with much of the difference explained by the couple who had one member in the NBU for the majority of the second 11 days. As the number of guests in quarantine decreased, this couple accounted for a greater percentage of the total population in quarantine.

\section{Guest Feedback}

A second source of data regarding the effectiveness of the Town Hall is feedback from guests, who provided both verbal and written comments. Regarding verbal feedback, the BHC asked guests on four occasions for their observations about what was helpful and what could be improved concerning the Town Hall. Feedback was solicited during the second (February 26, 2020), second-to-last (March 16, 2020), and two mid-quarantine (March 8 and March 10, 2020) Town Hall meetings, typically toward the end of the meeting and often in conjunction with selecting a topic for the subsequent meeting. In addition, guests periodically made spontaneous remarks about the Town Hall. Guests gave verbal permission for their comments to be included in ongoing quality improvement efforts and scientific publications. All feedback, whether solicited or spontaneous, was transcribed in real time by the BHC and is provided in Table 3 in the online supplemental material. In addition, written feedback was obtained from four guests who completed a guest experience survey prior to their departure. These four surveys were from guests who departed the NQU within nine days of the first Town Hall meeting, as the process for survey completion and collection was not sustainable beyond this time. ${ }^{6}$ The survey included two questions about the Town Hall. All responses to these two questions are included in the online

\footnotetext{
${ }^{6}$ Guest experience survey distribution and collection required the SAT team to identify guests scheduled for discharge the following day and provide a paper copy of the survey to an NQU nurse who then handed the survey to the guest when delivering one of their meals. On the next visit to the guest room, a nurse took a picture of the completed survey and emailed it to the BHC due to infection control protocols barring removal of items from quarantine rooms. With the changes in SAT team membership, the last-minute nature of departure for most guests in isolation (which depended on a third negative PCR result the day before), and the increasing burden on clinical staff as community patients were admitted to the NBU, no additional experience surveys were obtained after the initial four.
} 
supplemental material. Two additional sources of feedback were spontaneous comments made by guests during 1:1 consultation with the $\mathrm{BHC}$ and a letter sent by a guest after returning home, also included in the online supplemental material.

In all, 11 of the 15 guests (73\%) who were well enough to attend regularly provided feedback either during a Town Hall meeting, via the guest experience survey, in 1:1 interaction with the BHC, and/or in a letter. The author completed a semantic, inductive thematic analysis (Braun \& Clarke, 2006) of the transcribed feedback in which the data were reviewed for potential themes, then re-considered for fit with the themes in an iterative fashion until all comments fit one or more themes. In this manner, four main themes to the feedback were identified: 1) Helpfulness of hearing about others' experiences, 2) Gratitude for open communication with staff, 3) Benefits of learning stress management skills, and 4) Frustration with logistical challenges. ${ }^{7}$

\section{Helpfulness of Hearing About Others' Experiences}

Yalom and Leszcz (2005) identified "universality" as an elemental therapeutic factor in group psychotherapy, noting that "the disconfirmation of a client's feelings of uniqueness is a powerful source of relief" (p. 6). This sense of relief was reflected in Guest 3's experience survey, which noted that it was helpful "to hear how others were feeling or having some [of] the same feelings you are." Guest 9 expressed a similar sentiment during a Town Hall meeting, stating, "Connecting with everyone helps us not feel isolated like we're on an island." In a different meeting, Guest 1 likewise shared, "I am grateful for the Town Halls, to hear about how others are doing." Similarly, in 1:1 interaction with the BHC, Guest 11 stated that the Town Hall "is a good idea. It's a chance to hear how everyone is doing." These written and verbal comments suggest that guests appreciated the opportunity afforded by the Town Hall to

\footnotetext{
${ }^{7}$ At the suggestion of a reviewer, the feedback from the guests who had one or more individual meetings with the BHC was compared to that of the other guests. The comments of guests who had met individually with the BHC aligned closely with those of the larger group and fit easily within the identified themes, with no discernible differences.
} 
learn how the others in quarantine were feeling, which presumably provided some measure of validation for their own experiences and helped to decrease feelings of loneliness and isolation.

\section{Gratitude for Open Communication with Staff}

Several guests, including three of the four individuals who completed guest experience surveys, expressed appreciation for the open, clear and thorough communication with NQU doctors and staff. Responding to the survey prompt about which aspects of the Town Hall proved helpful, Guest 4 wrote, “1) Updates as to the testing schedule and the expectation as to how one leaves quarantine / isolation. 2) Opportunity to talk with the doctors and nurses to ask questions." Similarly, Guest 5 noted in their survey, "Update every day of the ongoing information of the COVID-19" and Guest 2 offered, "Doctors sharing real time CDC info." During a Town Hall meeting, Guest 2 also shared, "I appreciate the communication, which helps when you feel like you are stuck." Guest 6 commented, "These meetings are excellent. We are forced to stay here, it is not good psychologically, but the clear communication makes it a lot better." Guest 8 also expressed appreciation for the communication, declaring, "You are doing so well. You provide up to date information from the CDC. It's quite good for us. Outside people call me, and they consider me to have good knowledge." The feedback above indicates that the guests were grateful for the frequent, straightforward and detailed information from NQU/NBU physicians and staff as well as for the opportunity to ask questions.

\section{Benefits of Learning Stress Management Skills}

A third theme characterizing guest feedback was the benefits of learning stress management techniques. For example, when asked during a meeting what aspects of the Town Hall were helpful and what could be improved, Guest 8 answered, "Stress management and mindfulness. I liked that. Very helpful. Most people don't have access to that kind of knowledge." Guest 9 responded, "I'm open to any tools to help pass the time and keep positive. I need to learn to live for today and stay present and not worry about the future. I know stress is the worst thing for us with regard to our immune system, which 
we need to fight the virus." In addition, during a discussion of growth as a result of the quarantine experience, Guest 7 spontaneously offered, "I tried that exercise on the paper [a mindfulness technique involving counting breaths], and I fell asleep before I got to 10!" This was one of the only times this guest spoke during a Town Hall meeting.

Furthermore, after in-session mindfulness or relaxation exercises, guests commonly shared their experiences. Following a visualization exercise, for example, Guest 8 stated, "Brought me right back to [name of beach] where I used to go with my wife. It was so similar to being there. Very relaxing." After a body scan exercise, Guest 9 shared, "I always carry tension in my neck and shoulder for stress. This was helpful to relieve that a bit." Following this same activity, Guest 11 commented that it was "very relaxing." In addition, after an exercise that began with deep breathing, progressed to a body scan and ended with a mindfulness activity, Guest 1 shared that they became so relaxed that they fell asleep before the mindfulness portion.

Finally, Guest 8 sent a letter 12 days after release from quarantine updating the staff on their status and expressing gratitude for their care, stating, "I miss the town hall meeting and especially [the] wellness training. Those relaxing techniques are forever useful to everyone." Taken together, the comments suggest that many guests enjoyed the stress management exercises completed during the Town Hall meetings and may have used the techniques outside of the meetings as well.

\section{Frustration with Logistical Challenges}

The fourth theme characterizing guest feedback about the Town Hall meetings was logistical challenges. In response to the guest experience survey question about how the Town Hall could be improved, two guests commented on the miscommunication regarding the Zoom ID which caused confusion when guests called in to the second and third meetings. As a result of this error, some guests missed the February 26 and February 27 Town Halls, and those present experienced both delays and prolonged meetings as staff reached out to members individually to provide the correct ID. Guest 2 
wrote, "Shorter, on time, get phone \# [Zoom ID] for day's call straight before call," and Guest 5 noted, "The same phone number [Zoom ID] each time is helpful." A second logistical challenge identified by Guests 3 and 4 on the experience survey was occasional background noise during meetings, as one or two guests did not consistently mute their phones despite periodic reminders. (Guest 3 suggested, "Ask people to mute phone when not talking," and Guest 4 responded, "Providing guidance on how to mute the room phones.")

\section{Lessons Learned}

The National Quarantine Unit Town Hall was developed to mitigate the potentially negative psychological effects of a facility-based quarantine/isolation of indeterminate length following two weeks of quarantine on a cruise ship. Based on research concerning the psychological consequences of, and factors that increase stress during, quarantine and isolation, the specific objectives were to promote guest trust and confidence in staff by sharing timely and accurate information about COVID-19, address day-to-day needs and comfort on the unit, teach a range of behaviors and skills associated with individual resilience, and increase social support by both fostering a sense of cohesiveness and encouraging connection with outside supports. Guest verbal and written feedback addressed each of these objectives, reflecting appreciation for the high level of communication with staff, the value of learning stress management techniques, and the importance of hearing about others' experiences. The feedback, in conjunction with the high participation rate, suggests that the Town Hall was successful in meeting the objectives pertaining to trust and confidence in staff, addressing daily needs, and increasing skills associated with resilience. However, the feedback seems to indicate that the fourth objective, developing community and cohesiveness, was only partially met, as discussed below.

In one sense, the 15 cruise ship passengers formed a natural community. A recognition of common identity was reflected in guests' use of the pronouns "we" and "us" when providing feedback (e.g., "We are forced to stay here," "It's quite good for us," "Connecting with everyone helps us not feel isolated 
like we're on an island"), though it is also possible that such personal plural pronouns referred to the passenger and their spouse. Nonetheless, any sense of community-the result of finding themselves in a shared stressful circumstance-did not appear to evolve into group cohesiveness. Yalom and Leszcz (2005) describe cohesiveness as the attraction that members feel toward the group and one another, evidenced in mutual acceptance and support as well as development of meaningful relationships among members. While the feedback indicates that guests appreciated hearing about each other's experiences, consistent with Yalom and Leszc's "universality," it does not suggest that the participants grew closer to one another over time as one might expect (e.g., MacNair-Semands \& Lese, 2000). None of the comments, for example, reference relationships between members beyond guests' hearing about how others are faring. In addition, during Town Hall meetings, there was little spontaneous interaction among guests, who tended to remain quiet after their peers shared information.

Often, it fell to the BHC to invite guests to comment on observations, feelings and experiences shared by other guests. Even with role modeling and direct prompting by the BHC, guests often remained quiet or shared something new, but they rarely addressed peers or their verbalizations directly. In addition, on several occasions the BHC offered for guests to share their room telephone or cell phone numbers with the SAT team in order to develop a list for circulation to all guests, but only two guests provided a number. While group attendance was high, which in some circumstances can indicate cohesiveness (Yalom \& Leszcz, 2005), in this case it likely resulted from the value members placed on the content shared, including hearing about others' experiences in quarantine, rather than the group process itself. Stated differently, the sense of relief and validation in hearing that other Town Hall participants faced similar challenges did not appear to translate into trusting and supportive relationships.

As detailed by Yalom and Leszcz (2005), psychotherapy group leaders shape group norms-including expectations of members for providing feedback and support to one another-by establishing rules and 
modeling and reinforcing desired behaviors. Although the BHC employed these strategies in an attempt to foster mutual support among members, it is conceivable that a different group format or leadership style might have generated a higher degree of cohesiveness.

The Town Hall was designed to foster a sense of community and cohesiveness not only by establishing norms for feedback and support, but also by promoting several interdependent therapeutic factors underlying effective and cohesive psychotherapy groups, as outlined in Table 1. Beyond universality and cohesiveness, discussed above, these are altruism, instillation of hope, and imparting of information. The BHC attempted to generate altruism by providing opportunities for guests to help one another. While there were several occasions when guests shared advice-from where to stand to get better Wi-Fi reception to how to exercise in a small space - the extent of such assistance was limited, consistent with the overall reluctance to provide feedback to one another despite prompting by the $\mathrm{BHC}$, as described above.

With regard to instilling hope, the Town Hall ensured that guests heard examples of fellow passengers' managing the quarantine experience well and meeting criteria for discharge from the NQU. For example, when guests expressed frustration about how long it was taking to get three negative PCR tests (the criteria for release for the 13 SARS-CoV-2 positive passengers), the BHC pointed out that several passengers had already met the criteria and that those still in quarantine would follow suit. However, it was not clear whether guests internalized these messages and, thus, whether the intervention was successful in instilling hope. In contrast, the Town Hall did appear to be successful in imparting information, the third therapeutic factor identified above, as evidenced by expressions of appreciation for the medical information shared as well as comments indicating use of the stress management strategies taught.

It is an open question whether having a video component to the Town Hall might have promoted a greater sense of cohesiveness. Social psychology research suggests that while verbal information is more 
important than non-verbal information for empathic accuracy, having access to both types of information is superior (e.g., Zaki, Bolger \& Ochsner, 2009). In addition, studies conducted in a psychotherapy context suggest that nonverbal synchrony, or coordination of body movements, between therapist and patient is related to relationship quality and symptom reduction (Ramseyer \& Tschacher, 2011). Thus, the absence of visual contact may have inhibited the development of more meaningful relationships, and provision of audiovisual communication devices for future Town Hall quarantine efforts should be considered.

The failure to develop cohesiveness does not mean that the Town Hall was unsuccessful in the overarching goal of minimizing psychological distress among those in quarantine. While cohesiveness is important for progress in process-oriented group psychotherapy, the goal, objectives and structure of the Town Hall were different from those of group therapy. Indeed, the high participation rate, in concert with positive feedback regarding hearing about others' experiences, open communication with staff, and learning stress management skills, suggests that the intervention may have been successful in achieving most of its objectives, and holds promise for mitigating the potentially negative psychological effects of facility-based quarantine. A more definitive conclusion, however, awaits additional research.

\section{Future Directions}

While the psychological effects of quarantine have been the subject of numerous investigations, there has been considerably less focus on interventions designed to mitigate these effects. As the current pilot intervention was resource-intensive (daily 40- to 45-minute meetings with multiple staff members), a controlled trial based on the current model would be helpful in determining whether the expense is justified. If the Town Hall intervention is found to be effective, then treatment dismantling studies to establish which elements are necessary might be a valuable next step. In addition, research to determine the frequency of meetings needed to ameliorate psychological distress would be helpful, as the daily schedule employed in the current intervention may be more frequent than required to achieve 
the objectives. Investigating whether video teleconferencing improves outcomes over audio-only meetings is another area for further investigation. It would also be instructive to operationalize and measure the factors hypothesized to mediate the protective effects of the Town Hall, in particular, guest judgments of the trustworthiness and clarity of messages concerning the disease and infection control procedures, perceptions regarding adequacy of supplies, use of the strategies taught to support resilience, and perceived social support.

Most of the couples did not know one another, nor the local healthcare worker who joined the group in quarantine, prior to arriving at the NQU. In other settings, groups with established relationships, such as military units or disaster medical assistance teams (DMATs) returning from deployments, may be quarantined together. Research is necessary to determine how the current model might be adapted for such cohorts. For example, a Town Hall meeting for a DMAT in quarantine might include co-facilitation by a BHC and a DMAT leader. In addition, the Town Hall meeting might incorporate elements of existing DMAT meetings. Furthermore, because such groups may have already established a common identity and social connection, the Town Hall meetings might focus less on strategies to foster cohesion and more on information exchange and techniques associated with resilience. The model may also be useful when groups with established relationships, such as healthcare workers or first responders from the same institution or department, undergo home quarantine concurrently. Such work should consider the needs of families with children, who may be negatively impacted by disruptions in family routines and fear-inducing messages surrounding quarantine (Sprang \& Silman, 2013).

Future work with both pre-existing and newly-formed groups in quarantine might adopt the basic framework of the Town Hall (i.e., meetings for education and discussion with an interdisciplinary group of caregivers) but a different underlying model for psychosocial intervention. One such model is Psychological First Aid (PFA; Brymer et al., 2006), an evidence-informed intervention designed to 
decrease stress and promote positive coping in the aftermath of a traumatic event. PFA "core actions" (Brymer et al., 2006), which include safety and comfort, practical assistance, connection with social supports, and information on coping, are consistent with the objectives of the Town Hall. In addition, PFA training is widely available, including free online versions (e.g., https://learn.nctsn.org/course/index.php), and the skills can be learned by non-behavioral health providers. As a result, PFA's adoption within the current framework might allow for more rapid dissemination of the Town Hall model should controlled research demonstrate its effectiveness. A related strategy is training staff and guests, as well as family members, in community-based psychological first aid (CBPFA) during Town Halls meetings. CBPFA teaches regular citizens to provide mental health support to family, community members and themselves (Jacobs et al., 2016). This might be particularly well-suited for large-scale operations, such as New York City Health + Hospitals' isolation hotel program, which repurposed 700 hotel rooms to monitor residents who had suspected or confirmed COVID-19 with no safe place to quarantine (Jordan-Martin, et al., 2020).

\section{Limitations}

The NQU Town Hall was developed in real time as a component of the United States government's repatriation of 15 American citizens quarantined on a cruise ship off the coast of Japan due to an infectious disease outbreak. Consequently, the intervention was not part of a formal research protocol, and there was no control group nor baseline or outcome measures of psychological and adaptive functioning. While participation rate serves as one indicator of guest interest, it does not shed light on members' level of attention during discussions nor investment in the content. In addition, as discussed above, guest experience surveys were only provided to the first four guests released from quarantine due to logistical challenges; these guests may have had different experiences compared to those who stayed longer. Another significant limitation in evaluating the effectiveness of the Town Hall intervention is that feedback was not anonymous; rather, in many cases it was directly solicited by the 
BHC, which may have influenced participants to respond favorably. However, it should be noted that positive feedback was also provided spontaneously during Town Halls and 1:1 interaction as well as in a letter sent after release from quarantine. Moreover, the feedback had consistent themes, and participants offered criticism in addition to positive comments, suggesting that demand characteristics, if operational, were not so dominant that they inhibited all unfavorable feedback.

While heterogeneous with regard to race, ethnicity and state of residence, all participants, other than the local healthcare provider, were over the age of 50, married, and had the resources to take a cruise. The response of the current group to the Town Hall intervention may be different from those who are younger, single and less affluent. Furthermore, the small sample size limits generalizability, even to groups with demographic characteristics similar to those of the current cohort.

\section{Conclusions}

Quarantine and isolation are vital public health tools for mitigating the spread of HHCDs, but their use poses psychological risks, including symptoms of traumatic stress, depression and anxiety. The current Town Hall pilot intervention-designed to mitigate these potentially harmful psychological effects in a group of 16 individuals in quarantine/isolation at the National Quarantine Unit-provided timely and accurate information about the index disease, addressed concerns pertaining to basic needs and comfort, introduced skills associated with resilience, and attempted to foster a sense of community and cohesiveness. Verbal and written feedback from guests, in connection with high rates of participation, suggests that the intervention was successful in promoting trust in staff, meeting guests' needs for information, normalizing guest experiences, and providing tools to manage stress and enhance resilience. Given the likelihood of continued use of home- and facility-based quarantine as an infection control measure during the COVID-19 pandemic and future HHCD outbreaks, additional research is warranted, including randomized controlled trials based on the current model as well as adaptations for groups with established relationships, both in facility-based and home quarantine. 


\section{References}

Adler, A. B., Kim, P. K., Thomas, S. J., \& Sipos, M. L. (2018). Quarantine and the U.S. military response to the Ebola crisis: soldier health and attitudes. Public Health, 155, 95-98.

https://doi.org/10.1016/j.puhe.2017.11.020

Bai, Y., Lin, C., Lin, C., Chen, J., Chue, C., \& Chou, P. (2004). Survey of stress reactions among health care workers involved with the SARS outbreak. Psychiatric Services, 55(9), 1055-1057.

https://doi.org/10.1176/appi.ps.55.9.1055

Barratt, R., Shaban, R., Moyle, W. (2011). Behind barriers: Patients' perceptions of source isolation for Methicillin-resistant Staphylococcus aureus (MRSA). Australian Journal of Advanced Nursing, 28(2), 53-9.

Blendon, R. J., Benson, J. M., DesRoches, C. M., Raleigh, E., \& Taylor-Clark, K. (2004). The public's response to Severe Acute Respiratory Syndrome in Toronto and the United States. Clinical Infectious Diseases, 38, 925-931. https://doi.org/10.1086/382355

Braun, V., \& Clarke, V. (2006). Using thematic analysis in psychology. Qualitative Research in Psychology, 3, 77-101. https://doi.org/10.1191/1478088706qp063oa

Brooks, S. K., Webster, R. K., Smith, L. E., Woodland, L., Wessely, S., Greenberg, N., \& Rubin, G. J. (2020). The psychological impact of quarantine and how to reduce it: Rapid review of the evidence. Lancet, 395, 912-20. https://doi.org/10.1016/S0140-6736(20)30460-8

Brymer, M., Jacobs, A., Layne, C., Pynoos, R., Ruzek, J., Steinberg, A., Vernberg, E., \& Watson, P. (2006). Psychological first aid: Field operations guide ( $2^{\text {nd }}$ ed.). Washington, DC: National Child Traumatic Stress Network and National Center for PTSD.

Caleo, G., Duncombe, J., Jephcott, F., Lokuge, K., Mills, C., Looijen, E., Theoharaki, F., Kremer, R., Kleijer, K., Squire, J., Lamin, M., Stringer, B., Weiss, H. A., Culli, D., Di Tanna, G. L., \& Greig, J. (2018). The factors affecting household transmission dynamics and community compliance with Ebola control 
measures: a mixed-methods study in a rural village in Sierra Leone. BMC Public Health, 18:248. https://doi.org/10.1186/s12889-018-5158-6

Catalano, G., Houston, S. H., Catalano, M. C., Butera, A. S., Jennings. S. M., Hakala. S. M., Burrows, S. L., Hickey, M. G., Duss, C. V., Skelton, D.N., \& Laliotis, G. J. (2003). Anxiety and depression in hospitalized patients in resistant organism isolation. Southern Medical Journal, 96(2), 141-145. https://doi.org/10.1097/01.SMJ.0000050683.36014.2E

Cates, D. S., Gomes, P. G., \& Krasilovsky, A. M. (2018). Behavioral health support for patients, families, and healthcare workers. In A. Hewlett \& A. R. K. Murthy (Eds.), Bioemergency Planning: A Guide for Healthcare Facilities (pp. 195-214). Cham, Switzerland: Springer. https://doi.org/10.1007/978-3-319$\underline{77032-116}$

Cava, M. A., Fay, K. E., Beanlands, H. J., McCay, E. A., \& Wignall, R. (2005). The experience of quarantine for individuals affected by SARS in Toronto. Public Health Nursing, 22(5), 398-406. https://doi.org/10.1111/j.0737-1209.2005.220504.x

Centers for Disease Control and Prevention. (2020, August 12). COVID-19 Overview and infection prevention and control priorities in non-US healthcare settings.

https://www.cdc.gov/coronavirus/2019-ncov/hcp/non-us-settings/overview/index.html

Centers for Disease Control and Prevention (2003a). Use of quarantine to prevent transmission of Severe Acute Respiratory Syndrome - Taiwan. Morbidity and Mortality Weekly Report, 52(29), 680683. https://www.cdc.gov/mmwr/preview/mmwrhtml/mm5229a2.htm

Centers for Disease Control and Prevention (2003b). Efficiency of quarantine during an epidemic of Severe Acute Respiratory Syndrome - Beijing, China, 2003, Morbidity and Mortality Weekly Report, 52(43), 1037-1040. https://www.cdc.gov/mmwr/preview/mmwrhtml/mm5243a2.htm

Coronavirus Resource Center (2020). Mortality Analysis. Johns Hopkins University. Retrieved October 30, 2020, from https://coronavirus.jhu.edu/data/mortality 
Day, H. R., Morgan, D. J., Himelhoch, S., Young, A., \& Perencevich, E. N. (2011). Association between depression and contact precautions in veterans at hospital admission. American Journal of Infection Control, 39(2), 163-165. https://doi.org/10.1016/j.ajic.2010.06.024

Day. H. R., Perencevich, E. N., Harris, A. D., Gruber-Baldini, A. L., Himelhoch, S. S., Brown, C. H., \& Morgan, D. J. (2013). Depression, anxiety, and moods of hospitalized patients under contact precautions. Infection Control and Hospital Epidemiology, 34(3), 251-258. https://doi.org/10.1086/669526

Desclaux, A., Badji, D., Ndione, A. G., \& Sow, K. (2017). Accepted monitoring or endured quarantine? Ebola contacts' perceptions in Senegal. Social Science and Medicine, 178, 38-45. https://doi.org/10.1016/j.socscimed.2017.02.009

DiGiovanni, C., Conley, J., Chiu, D., \& Zaborski, J. (2004). Factors influencing compliance with quarantine in Toronto during the 2003 SARS outbreak. Biosecurity and Bioterrorism: Biodefense Strategy, Practice and Science, 2(4), 265-272. https://www.liebertpub.com/doi/10.1089/bsp.2004.2.265

Gammon, J. (1998). Analysis of the stressful effects of hospitalisation and source isolation on coping and psychological constructs. International Journal of Nursing Practice, 4, 84-96. https://doi.org/10.1046/i.1440-172X.1998.00084.x

Goyal, M., Singh, S., Sibinga, E. M. S., Gould, N. F., Rowland-Seymour, A., Sharma, R., Berger, Z., Sleicher, D., Maron, D. D., Shihab, H. M., Ranasinghe, P. D., Linn, S., Saha, S., Bass, E. B., \& Haythornthwaite, J. A. (2014). Meditation programs for psychological stress and well-being: A systematic review and metaanalysis. JAMA Internal Medicine, 174(3), 357-368.

https://doi.org/10.1001/jamainternmed.2013.13018

Guillemin, I., Marrel, A., Lambert, J., Beriot-Mathiot, A., Doucet, C., Kazoglou, O., Luxemburger, C., Reygrobellet, C., \& Arnould, B. (2014). Patients' experience and perception of hospital-treated 
Clostridium difficile infections: a qualitative study. The Patient-Patient-Centered Outcomes Research, 7, 97-105. https://doi.org/10.1007/s40271-013-0043-y

Hawryluck, L., Gold, W. L., Robinson, S., Pogorski, S., Galea, S., \& Styra, R. (2004). SARS Control and Psychological Effects of Quarantine, Toronto, Canada. Emerging Infectious Diseases, 10(7), 1206-1212. https://www.ncbi.nlm.nih.gov/pmc/articles/PMC3323345

Holt-Lunstad, J., Robles, T. F., \& Sbarra, D.A. (2017). Advancing social connection as a public health priority in the Unites States, American Psychologist, 72(6), 517-530.

http://dx.doi.org/10.1037/amp0000103

Jacobs, G. A., Gray, B. L., Erickson, S. E., Gonzalez, E. D., \& Quevillon, R. P. (2016). Disaster mental health and community-based psychological first aid: Concepts and education/training. Journal of Clinical Psychology, 72, 1307-1317. https://doi.org/10.1002/jclp.22316

Jordan-Martin, N. C., Madad, S., Alves, L., Wang, J., O’Gere, L., Smith, Y. G., Pressman, M., Shure, J. A., \& Cosmi, M. (2020). Isolation hotels: A community-based intervention to mitigate the spread of the COVID-19 pandemic. Health Security, 18 (5). https://doi.org/10.1089/hs.2020.0123

Jeong, H., Yim, H. W., Song, Y., Ki, M., Min, J., Cho, J., \& Chae, J. (2016). Mental health status of people isolated due to Middle East Respiratory Syndrome. Epidemiology and Health, 38, e2016048. https://doi.org/10.4178/epih.e2016048

Kim, H., \& Kim, E. J. (2018). Effects of relaxation therapy on anxiety disorders: A systematic review and meta-analysis. Archives of Psychiatric Nursing, 32(2), 278-284.

https://doi.org/10.1016/j.apnu.2017.11.015

Knowles, H. (1993). The experience of infectious patients in isolation. Nursing Times, 89(30), 53-56.

Li, M., Wang, L., Jiang, M., Wu, D., Tian, T., \& Huang, W. (2020). Relaxation techniques for depressive disorders in adults: a systematic review and meta-analysis of randomised controlled trials. 
International Journal of Psychiatry in Clinical Practice.

https://doi.org/10.1080/13651501.2020.1764587

Liu, X., Kakade, M., Fuller, C. J., Fan, B., Fang, Y., Kong, J., Guan, Z., \& Wu, P. (2012). Depression after exposure to stressful events: Lessons learned from the severe acute respiratory syndrome epidemic. Comprehensive Psychiatry, 53, 15-23. https://doi.org/10.1016/i.comppsych.2011.02.003

MacNair-Semands, R. R., \& Leese, K. P. (2000). Interpersonal problems and the perception of therapeutic factors in group therapy. Small Group Research, 31(2), 158-174.

Madeo M. (2001). Understanding the MRSA experience. Nursing Times, 97(30), 36-37.

https://www.nursingtimes.net/clinical-archive/understanding-the-mrsa-experience-26-07-2001

Masten, A. (2014). Global perspectives on resilience in children and youth. Child Development, 85(1), 6-

20. https://doi.org/10.1111/cdev.12205

Mayho, P. (1999). Barrier grief. Nursing Times, 95(31), 24-25.

McMillan, D. W., \& Chavis, D. M. (1986). Sense of community: A definition and theory. Journal of

Community Psychology, 14, 6-23. https://psycnet.apa.org/doi/10.1002/1520-

6629(198601)14:1\%3C6::AID-JCOP2290140103\%3E3.0.CO;2-I

Meichenbaum, D. (2012). Roadmap to Resilience: A Guide for Military, Trauma Victims and Their

Families. Belleair, FL: Institute Press.

Newton, J. T., Constable, D., \& Senior, V. (2001). Patients' perceptions of methicillin-resistant

Staphylococcus aureus and source isolation: a qualitative analysis of source-isolated patients. Journal of Hospital Infection, 48, 275-280. https://doi.org/10.1053/ihin.2001.1019

New York City Department of Health and Mental Hygiene. (2010). Adaptable Quarantine Procedures Manual. Unpublished manual.

Oldman T. (1998). Isolated cases. Nursing Times, 94(11), 67-70. 
Pan, P. J. D., Chang, S., \& Yu, Y. (2005). A support group for home-quarantined college students exposed to SARS: Learning from practice. The Journal for Specialists in Group Work, 30(4), 363-374. https://doi.org/10.1080/01933920500186951

Park, E., Baim, P., Kagan, L., \& Buchanan. (2015). Stress Management and Resiliency Training: The Relaxation Response Resiliency Program Manual. Boston: Benson-Henry Institute for Mind Body Medicine at Massachusetts General Hospital.

Ramseyer, F., \& Tschacher, W. (2011). Nonverbal synchrony in psychotherapy: Coordinated body movement reflects relationship quality and outcome. Journal of Consulting and Clinical Psychology, 79(3), 284-295. https://doi.org/10.1037/a0023419

Reynolds, D. L., Garay, J. R., Deamond, S. L., Moran, M. K., Gold, W., \& Styra, R. (2008). Understanding, compliance and psychological impact of the SARS quarantine experience. Epidemiology and Infection, 136(7), 997-1007. https://doi.org/10.1017/S0950268807009156

Skyman, E., Sjostrom, H. T., \& Hellstrom, L. (2010). Patients' experiences of being infected with MRSA at a hospital and subsequently source isolated. Scandinavian Journal of Caring Sciences, 24, 101-107. https://doi.org/10.1111/j.1471-6712.2009.00692.x

Soon, M. M. L., Madigan, E., Jones, K. R., \& Salata, R. A. (2013). An exploration of the psychologic impact of contact isolation on patients in Singapore. American Journal of Infection Control, 41(10), e111-e113. http://dx.doi.org/10.1016/j.ajic.2013.01.037

Southwick, S. M., Bonanno, G. A., Masten, A. S., Panter-Brick, C., \& Yehuda, R. (2014). Resilience definitions, theory, and challenges: Interdisciplinary perspectives. European Journal of Psychotraumatology, 5(1), 25338. https://doi.org/10.3402/ejpt.v5.25338

Sprang, G., \& Silman, M. (2013). Posttraumatic stress disorder in parents and youth after health-related disasters. Disaster Medicine and Public Health Preparedness, 7, 105-110.

https://doi.org/10.1017/dmp.2013.22 
Tarzi, S., Kennedy, P., Stone, S., \& Evans, M. (2001). Methicillin-resistant Staphylococcus aureus:

Psychological impact of hospitalization and isolation in an older adult population. Journal of Hospital Infection, 49(4), 250-254. https://doi.org/10.1053/jhin.2001.1098

Walsh, R. (2011). Lifestyle and mental health. American Psychologist, 66(7), 579-592. https://doi.org/10.1037/a0021769

Ward, D. (2000). Infection control: Reducing the psychological effects of isolation. British Journal of Nursing, 9(3), 162-70. https://doi.org/10.12968/bjon.2000.9.3.162

Wu, P., Fang, Y., Guan, Z., Fan, B., Kong, J., Yao, Z., Liu, X., Fuller, C., Susser, E., Lu, J., Hoven, C. (2009). The psychological impact of the SARS epidemic on hospital employees in China: Exposure, risk perception, and altruistic acceptance of risk. Canadian Journal of Psychiatry, 54(5), 302-311. https://doi.org/10.1177/070674370905400504

Yalom, I. D., \& Leszcz, M. (2005). The Theory and Practice of Group Psychotherapy (5 ${ }^{\text {th }}$ ed.). New York: Basic Books.

Zaki, J., Bolger, N., \& Ochsner, K. (2009). Unpacking the informational bases of empathic accuracy. Emotion, 9(4), 478-487. https://doi.org/10.1037/a0016551

Zech, N., Hansen, E., Bernardy, K., \& Hauser, W. (2017). Efficacy, acceptability and safety of guided imagery/hypnosis in fibromyalgia - A systematic review and meta-analysis of randomized controlled trials. European Journal of Pain, 21(2), 217-227. https://doi.org/doi:10.1002/ejp.933 


\section{Table 1}

Therapeutic Factors and Associated Town Hall Interventions

Therapeutic factor Town Hall intervention

(Yalom \& Leszcz, 2005)

Universality

Promote sharing of information among guests to help them discover that others may be experiencing the same thoughts and feelings.

Altruism

Give guests a chance to help one another in order to increase their selfesteem and sense of purpose.

Instillation of hope

Allow guests to hear that others are managing the experience well and are meeting criteria for release from quarantine.

Imparting of information Provide verbal and written descriptions of expected psychological reactions to the quarantine experience and return home, and teach a variety of strategies believed to contribute to resilience.

Group cohesiveness

Encourage guests to interact with and provide feedback to one another both within and outside of Town Hall meetings. 


\section{Figure 1}

Town Hall Participation (February 25, 2020 through March 18, 2020)

Town Hall Participation

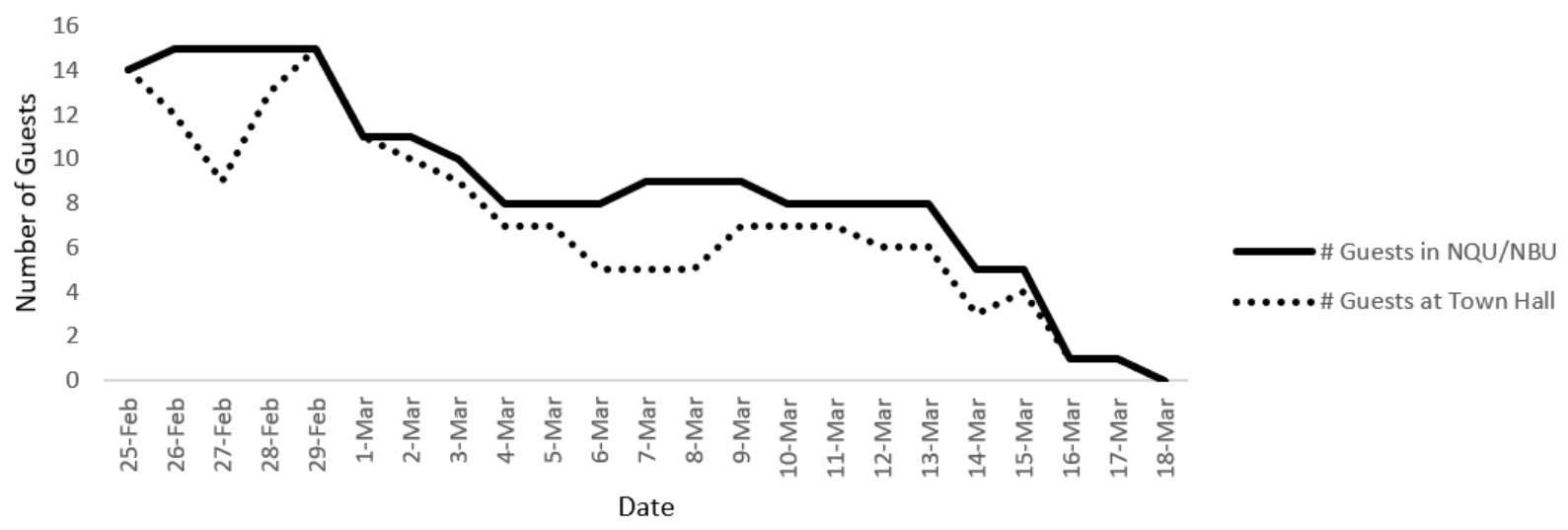

